\title{
Efectos gammágenos del cobre en los aceros inoxidables $18 \mathrm{Cr} 8 \mathrm{Ni}^{(\bullet)}$
}

\author{
J. Botella $^{(*)}$, P. Hierro ${ }^{(* *)}$ y C. Merino ${ }^{(* *)}$ \\ Resumen A partir de una serie de 22 lingotes de $40 \mathrm{~kg}$ de aceros inoxidables típicos $18 \mathrm{Cr} 8 \mathrm{Ni}$, con concentra- \\ ciones variables de cobre entre 0,6 y $3,0 \%$ en masa, se mide la ferrita $\delta$ mediante un medidor de \\ ferrita y se deduce para el cobre un equivalente en níquel de 0,27 , a la vez que se establecen ciertas \\ diferencias entre los contenidos de ferrita $\delta$ medida y la deducida según DeLong -excluida la acción \\ gammágena del cobre- por el hecho de solidificar en lingotes en vez de la típica solidificación de sol- \\ daduras.
}

Palabras clave: Aceros inoxidables austeníticos. Ferrita $\delta$. Equivalente en níquel del cobre.

\section{Gamma-genes effects of the copper in the $18 \mathrm{Cr} 8 \mathrm{Ni}$ stainless steels}

\begin{abstract}
From a series of 22 typical $18 \mathrm{Cr} 8 \mathrm{Ni}$ stainless steel $40 \mathrm{~kg}$ ingots, with copper variable concentrations from 0.6 to 3.0 weight $\%, \delta$-ferrite is measured with a ferrite-meter device, calculating a nickel equivalent of 0.27 for copper. Some differences between the $\delta$-ferrite and that on calculated by DeLong -excluding the copper $\gamma$-gene action- have been found because of different solidification and cooling regimes in ingot and weld cases.
\end{abstract}

Keywords: Austenitic stainless steels. $\delta$-ferrite. Nickel equivalent of the copper.

\section{INTRODUCCIÓN}

El uso del cobre como aleante en la fabricación de los aceros inoxidables o su presencia involuntaria, debido al empleo como materias primas de chatarras contaminadas, ha sido siempre un tema controvertido entre los aceristas ya que dicho elemento proporciona ciertas ventajas frente a posibles inconvenientes.

Quizás el único inconveniente citado tradicionalmente es el deterioro de la ductilidad en caliente, imputándosele al cobre la formación de grietas en los bordes de las bobinas laminadas en caliente (edge cracks) y astillados superficiales (slivers). Investigaciones previas (1) han conseguido cuantificar el deterioro relativo de la ductilidad en caliente provocado por el cobre y por otros elementos de aleación en el acero $18 \mathrm{Cr} 8 \mathrm{Ni}$, poniendo de manifiesto que el efecto de cantidades discretas de cobre -incluso hasta el $2 \%$

(•) Trabajo recibido el día 19 de noviembre de 1996

(*) Acerinox, S.A. Centro de Investigaciones y Ensayos. Los Palmones. Los Barrios. 11379-Algeciras. Cádiz (España).

(**) Dpto. de Ciencias de los Materiales e Ingeniería Metalúrgica. Facultad de Ciencias Químicas. Universidad Complutense. 28040-Madrid.
$\mathrm{Cu}$ en masa- es prácticamente irrelevante a ciertas temperaturas y siempre que se eviten los efectos sinérgicos negativos producidos por otros elementos contaminantes, como se resume en la tabla I.

TABLA I.- Ductilidad en caliente del acero inoxidable $18 \mathrm{Cr} 8 \mathrm{Ni}$ como función de algunos elementos químicos

TABLE I.- 18Cr8Ni hot ductility as a function of some chemical elements

\begin{tabular}{|c|c|}
\hline$T,{ }^{\circ} \mathrm{C}$ & Ductilidad en caliente $^{(*)}$ \\
\hline 1.000 & $\begin{array}{l}\text { \% R.A. }=67,2-15,5 \mathrm{P}-6,6(\mathrm{Cu})- \\
-43,7 \mathrm{As}-136,4 \mathrm{Sn}\end{array}$ \\
\hline 1.100 & $\begin{array}{l}\text { \% R.A. }=77,2-11,6 \mathrm{P}-2,0(\mathrm{Cu})- \\
-20,9 \mathrm{As}-48,0 \mathrm{Sn}\end{array}$ \\
\hline 1.200 & $\begin{array}{l}\text { \% R.A. }=91,0-37,1 \mathrm{P}-1,9(\mathrm{Cu})- \\
-6,4 \mathrm{As}-41,8 \mathrm{Sn}\end{array}$ \\
\hline 1.300 & $\begin{array}{l}\text { \% R.A. }=98,3-139,8 \mathrm{P}-11,4(\mathrm{Cu})- \\
-125,5 \mathrm{As}-176,6 \mathrm{Sn}\end{array}$ \\
\hline
\end{tabular}

(*) Expresada como \% R.A. (porcentaje de reducción de área en rotura de probeta cilíndrica de $10 \mathrm{~mm}$ de diámetro, a tracción). Elementos expresados en tanto por ciento en masa. 
A pesar de este inconveniente, es conocido el papel que juega la aportación de pequeñas cantidades de cobre como promotor y estabilizante de la austenita, como elemento que mejora el comportamiento frente a la corrosión en ciertos medios, como retardador del endurecimiento en la deformación en frío, como controlador de propiedades mecánicas -dureza, límite elástico, resistencia a la tracción- imprimiendo una extraordinaria calidad de pulido en cubertería y orfebrería, por citar algunas de las ventajas.

En la acción estabilizante de la austenita frente a la transformación martensítica por deformación aparece el cobre con una eficacia el doble que la del níquel o que la del manganeso, por ejemplo, y superior a la del cromo en la disminución de la temperatura a la que, para una deformación verdadera del $30 \%$ se genera un $50 \%$ de martensita, como se deduce de la ecuación derivada de la de Ángel (2):

$$
\begin{aligned}
& \operatorname{Md}_{\left(\alpha^{\prime}\right)}(30,50)=413-9,5 \mathrm{Ni}-13,7 \mathrm{Cr}-8,1 \mathrm{Mn}- \\
& 9,2 \mathrm{Si}-18,5 \mathrm{Mo}-17,1 \mathrm{Cu}-462(\mathrm{C}+\mathrm{N})
\end{aligned}
$$

Hull (3) encuentra relaciones diferentes, aunque también van en el sentido de mayor potencia del cobre que del níquel, manganeso y cromo, frente a la formación de martensita por deformación.

Otro tanto ocurre con el poder del cobre como estabilizante de la austenita frente a la transformación martensítica por efecto de la variación de temperatura. Aunque Eichelmann y Hull (4) no trabajaron con austenitas metaestables aleadas con cobre para encontrar la expresión que relaciona la composición química -en tanto por ciento en masa- con la temperatura -en grados centígrados- del inicio de la transformación martensítica:

$$
\mathrm{Ms}_{\left(\alpha^{\prime}\right)} 1305-61,1 \mathrm{Ni}-41,7 \mathrm{Cr}-33,3 \mathrm{Mn}-27,8 \mathrm{Si}-
$$

fue el propio Hull (3) quien, más tarde, estableció una potencia estabilizadora del cobre superior a las del níquel, manganeso, cromo y molibdeno.

Los trabajos de Ohkubo y col. (5), con austenitas estables típicas del acero $17 \mathrm{Cr} 12 \mathrm{Ni}$, cuantifican efectos del cobre sobre las propiedades mecánicas, con validez hasta contenidos de $\leq 2 \% \mathrm{Cu}$ en masa:

$$
\begin{gathered}
\mathrm{HV}_{(10)}=8,7 \times 10 \mathrm{C}+7,9 \times 10^{2} \mathrm{~N}+2,0 \mathrm{Si}+2,7 \mathrm{Cr}+ \\
10 \mathrm{Mo}-6,7 \mathrm{Ni}-2,6 \mathrm{Cu}+1,3 \times 10^{2} \\
\sigma_{0,2}\left(\mathrm{~N} / \mathrm{mm}^{2}\right)=3,5 \times 10^{2} \mathrm{C}+6,5 \times 10^{2} \mathrm{~N}+2,0 \times \\
10 \mathrm{Si}+2,0 \times 10 \mathrm{Cr}+7,3 \mathrm{Mo}-0,3 \mathrm{Mn}-4,2 \mathrm{Ni}- \\
5,6 \mathrm{Cu}-1,1 \times 10^{2}
\end{gathered}
$$

$\sigma_{\mathrm{B}}\left(\mathrm{N} / \mathrm{mm}^{2}\right)=1,1 \times 10^{3} \mathrm{C}+9,8 \times 10^{2} \mathrm{~N}+4,9 \mathrm{Si}+$ $1,3 \times 10 \mathrm{Cr}+1,5 \times 10 \mathrm{Mo}-0,3 \mathrm{Mn}-1,8 \times 10 \mathrm{Ni}$ -

$$
1,4 \times 10 \mathrm{Cu}+5,0 \times 10^{2}
$$

En cuanto a los efectos promotores de la austenita, disminuyendo el contenido de ferrita $\delta$ de solidificación en los aceros inoxidables típicos de la serie 300 de AISI, varios autores $(3,6$ y 7$)$ ofrecen para el cobre equivalentes en níquel que van desde el 0,30 al 0,51 . Si a los importantes efectos revisados con anterioridad se le une esta posibilidad de poder substituir una parte del costoso níquel de la aleación, consiguiendo un ahorro importante en el precio de las materias primas, se puede concluir que está más que justificado el estudio del empleo del cobre en la metalurgia del acero inoxidable. El trabajo que se presenta es una aportación en este sentido.

\section{MATERIALES Y MÉTODOS EXPERIMENTALES}

Mediante el empleo de un horno de inducción de laboratorio se han fabricado 22 lingotes de $40 \mathrm{~kg}$ de peso cada uno, con una composición típica $18 \mathrm{Cr} 8 \mathrm{Ni}$ y con concentraciones variables entre 0,6 y $3,0 \% \mathrm{Cu}$ en masa. Para una composición química determinada, el porcentaje de ferrita $\delta$ va a depender de las condiciones de solidificación y del enfriamiento posterior. Así pues, en la fabricación de los lingotes se ha procurado que, tanto las condiciones de solidificación como las del enfriamiento fuesen una invariable. La temperatura de colada se ha mantenido en el rango restrictivo de $1.550 \pm 5$ ${ }^{\circ} \mathrm{C}$, la velocidad de colada (volcado crisol-lingotera) fue normalizada a 2 min de tiempo total; en todos los casos se empleó la misma geometría y los mismos materiales en la lingotera y en la placa de lingotera, siguiéndose siempre, una vez colado el lingote, el mismo procedimiento de enfriamiento del conjunto lingotera-placa-lingote.

Los lingotes, cortados según muestra la figura 1 , se analizaron químicamente y se midieron los contenidos de ferrita $\delta$ mediante un medidor Förster 1053, contrastado con un equipo Magne Gage, en las secciones $\mathrm{AA}_{1} \mathrm{~A}_{2} \mathrm{~A}_{3}$ y $\mathrm{BB}_{1} \mathrm{~B}_{2} \mathrm{~B}_{3}$ señaladas en la figura, tomando 20 medidas por cada sección, cinco por cada lado, en puntos situados a unos $20 \mathrm{~mm}$ de los bordes, promediando los valores obtenidos para definir el contenido de ferrita $\delta$ de cada material.

\section{RESULTADOS OBTENIDOS}

La tabla II presenta las composiciones químicas obtenidas y los equivalentes en cromo y en níquel calculados según DeLong, así como los valores porcentuales de ferrita $\delta\left(\% \Delta_{\mathrm{D}}\right)$ obtenidos a partir del diagrama de constitución según DeLong, empleando los siguientes equivalentes: 


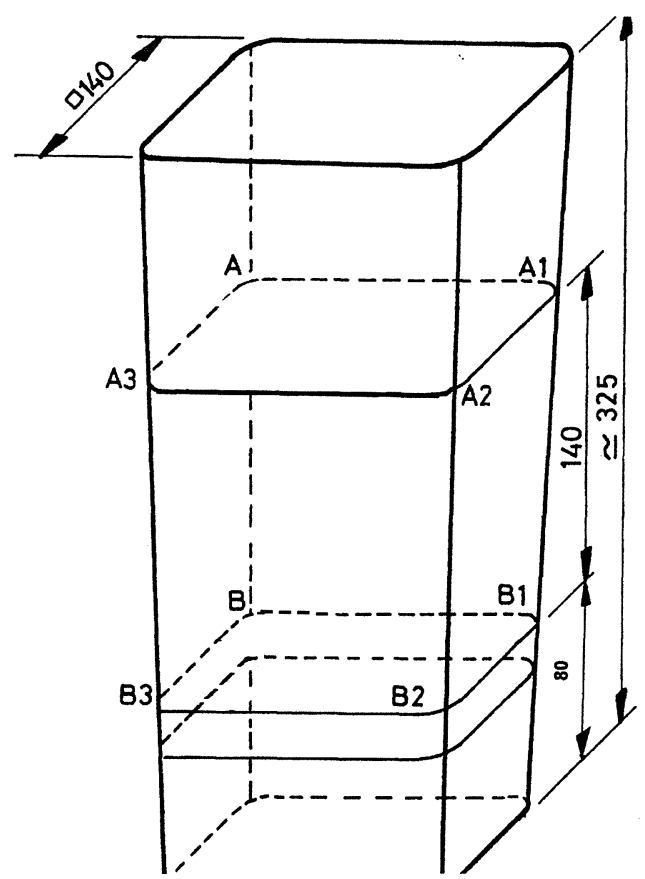

Fig. 1.- Croquis del lingote de $40 \mathrm{~kg}(\mathrm{~mm})$. Medidas de ferrita $\delta$ en secciones $\mathrm{AA}_{1} \mathrm{~A}_{2} \mathrm{~A}_{3}$ y $\mathrm{BB}_{1} \mathrm{~B}_{2} \mathrm{~B}_{3}$.

FIG. 1.- $40 \mathrm{~kg}$ ingot sketch $(\mathrm{mm}) \delta$ ferrite measured in $A A_{1} A_{2} A_{3}$ and $B B_{1} B_{2} B_{3}$ sections.

$$
\begin{gathered}
\mathrm{Cr}_{\text {equi }}=(\% \mathrm{Cr})+(\% \mathrm{Mo})+1,5(\% \mathrm{Si}) \\
\mathrm{Ni}_{\text {equi }}=(\% \mathrm{Ni})+\underset{(\% \mathrm{Mn})}{30[(\% \mathrm{C})+(\% \mathrm{~N})]+0,50} \\
\% \Delta_{\mathrm{D}}=\left[\frac{\mathrm{Cr}_{\text {equi }}+18}{\mathrm{Ni}_{\text {equi }}+36}+0,262\right] 161-160,65
\end{gathered}
$$

La tabla III presenta valores estadísticos de los elementos de composición de todos los lingotes, a excepción del cobre, poniéndose de manifiesto la relativa poca variación de dichos elementos, y dentro de un objetivo obvio de que las composiciones químicas no rindiesen materiales austeníticos monofásicos en los que fuese imposible evaluar el efecto gammágeno del cobre.

Volviendo a la tabla II, aparecen en ella los valores promedio obtenidos de las medidas en los lingotes de la ferrita $\delta\left(\% \Delta_{\mathrm{m}}\right)$ empleando el equipo Förster.

En otra columna de la tabla II aparecen las diferencias $\left(\% \Delta_{\mathrm{D}}\right)$ - $\left(\% \Delta_{\mathrm{m}}\right)$ entre los valores de ferrita $\delta$ predichos según el diagrama de constitución de DeLong y los valores de ferrita $\delta$ medidos en los lingotes. En la figura 2 se muestra la variación de estas diferencias en función de las concentraciones de cobre. Dos de las parejas de valores han sido descartadas aplicando criterios estadísticos. Puede observarse que el aumento de la concentración de cobre implica un aumento lineal entre la diferencia de la cantidad de ferrita $\delta$ predicha según DeLong y la calculada experimentalmente $\left(\% \Delta_{\mathrm{D}}\right)$ - $\left(\% \Delta_{\mathrm{m}}\right)$.

A partir de estos resultados, es posible proponer una primera tentativa de cálculo del equivalente en níquel del cobre. Efectivamente, a medida que aumenta el contenido de cobre, la medida de ferrita $\delta$ va siendo cada vez menor con respecto a la predicha por DeLong.

Esta disminución de la ferrita $\delta$ con la concentración de cobre viene expresada matemáticamente por el coeficiente angular de la recta:

$$
\left(\% \Delta_{\mathrm{D}}\right)-\left(\% \Delta_{\mathrm{m}}\right)=0,89+0,72(\% \mathrm{Cu})
$$

Un aumento del $1 \% \mathrm{Cu}$ producirá una disminución de $0,72 \%$ de ferrita $\delta$ en el lingote. La cuestión, ahora, es poder evaluar el efecto que el aumento del $1 \%$ Ni producirá sobre la disminución de ferrita en el lingote, de cara a definir un eventual equivalente en níquel del cobre.

Partiendo de la composición promedio que aparece en la tabla III -excluido el cobre- de los lingotes de la población estudiada, se calculan los equivalentes en cromo y níquel de dicha composición promedio:

$$
\begin{gathered}
\mathrm{Cr}_{\text {equi }}=18,28+0,20+1,5(0,34)=18,99 \\
\mathrm{Ni}_{\text {equi }}=8,23+30[(0,057)+(0,0428)]+0,5 \\
(1,40)=11,93
\end{gathered}
$$

Con estos datos, se deduce que el lingote básico tendrá, según DeLong, un $\Delta_{\mathrm{D}}=5,78 \%$.

Un incremento del $1 \% \mathrm{Ni}$ supondría un nuevo valor de ferrita $\delta$ del $3,24 \%$, por lo que la disminución de ferrita $\delta$ para un incremento de $1 \%$ Ni sería de $2,54 \%$. El equivalente en níquel o potencia gammágena del cobre, vendrá dada, en primera aproximación, por la relación de potencias gammágenas de cobre y níquel:

Equivalente en níquel del cobre $=\frac{0,72}{2,54}=0,28$

Volviendo a la figura 2 ó a la ec. [13], resulta que existe una ordenada en el origen bien definida, de modo que para una concentración nula de cobre,

$$
\left(\% \Delta_{\mathrm{D}}\right)-\left(\% \Delta_{\mathrm{m}}\right)=0,89
$$

A este hecho se le puede dar el significado de que, cuando se mide ferrita $\delta$ en los lingotes de 40 $\mathrm{kg}$, con independencia del efecto del cobre, se encuentran valores en cantidades $0,89 \%$ inferiores a los predichos por DeLong para un material solidificado como soldadura. Las razones pudieran estar en las diferencias de solidificación y enfriamiento entre una soldadura y un lingote (en estas condiciones 
TABLA II.- Composición química (\% en masa) y ferrita $\delta$ medida en los lingotes. Parámetros de cálculo para la determinación del equivalente en níquel del cobre

TABLE II.- Chemical composition (weight \%) and measured $\delta$ ferrite of the ingots. Calculated parameters to determine nickel equivalent of copper

\begin{tabular}{|c|c|c|c|c|c|c|c|c|c|c|c|c|c|c|c|c|}
\hline & \multicolumn{8}{|c|}{ Composición química, \% en masa } & \multirow[b]{2}{*}{$\mathrm{Cr}_{\text {equi }}$} & \multirow[b]{2}{*}{$\mathrm{Ni}_{\text {equi }}$} & \multirow[b]{2}{*}{$\Delta_{\mathrm{D}}$} & \multirow[b]{2}{*}{$\Delta_{\mathrm{m}}$} & \multirow[b]{2}{*}{$\Delta_{\mathrm{D}}-\Delta_{\mathrm{m}}$} & \multirow[b]{2}{*}{$\begin{array}{c}\Delta_{\mathrm{m}}+ \\
0,89\end{array}$} & \multirow[b]{2}{*}{$h^{*}$} & \multirow[b]{2}{*}{$\Delta_{\mathrm{D}}^{\prime}}$, \\
\hline Lingote & $\mathrm{C}$ & $\mathrm{Si}$ & $\mathrm{Mn}$ & $\mathrm{Ni}$ & $\mathrm{Cr}$ & Mo & $\mathrm{N}$ & $\mathrm{Cu}$ & & & & & & & & \\
\hline 1 & 0,052 & 0,34 & 1,42 & 8,24 & 18,31 & 0,20 & 0,0431 & 3,02 & 19,02 & 11,80 & 6,22 & 3,08 & 3,14 & 3,97 & 0,29 & 4,12 \\
\hline 2 & & 0,29 & 1, & & & & & 2,83 & & & & 90 & & & & \\
\hline 3 & 053 & 0,35 & 1 , & & 27 & & & 2,02 & 18 & & 99 & 3,58 & & 47 & 30 & 4,58 \\
\hline 4 & 61 & 0,21 & 1, & & 20 & & 0438 & 2,02 & & & & & & & 0,28 & 3,15 \\
\hline 5 & & 0,33 & 1 , & & & & 78 & 2,01 & & & & & & & & 3,79 \\
\hline 6 & & 0,30 & 1, & & & & & 1,98 & & & & & & & & 5,05 \\
\hline 7 & & 0,36 & 1,3 & 24 & & 21 & 0,0 & 1,96 & & & & 3,30 & & & 0,35 & 4,58 \\
\hline 8 & 056 & 0,30 & 1,40 & 8,02 & & & 11 & 1,96 & 18 & 11 &, 40 & 2,93 & & & 0,12 & 3,05 \\
\hline 9 & 054 & 0,35 & 1,45 & 8,73 & & & & 1,55 & & & ,34 & 3,20 & & 09 & 0,32 & 4,27 \\
\hline 10 & 055 & 0,33 & 1,44 & 8,19 & & 19 & 0,04 & 1,49 & & &, 14 & 3,90 & & & 0,35 & 5,10 \\
\hline 11 & ,053 & 0,39 & 1,27 & 8,22 & & 20 & 0,0 & 0,97 & & & 81 & 4,92 & & & 0,40 & 6,14 \\
\hline 12 & 0,057 & 0,34 & 1,40 & & & 0,18 & 0,0400 & 0,95 & & & & 5,1 & & & 0,12 & 5,67 \\
\hline 13 & 0,058 & 0,34 & 1,45 & & & & 0,0424 & 0,94 & 18,90 & & & 4,0 & & & 0,27 & 4,92 \\
\hline 14 & 0,059 & 0,38 & 1,4 & & & & & 0,93 & & & & 4,01 & & & 0,27 & 4,90 \\
\hline 15 & 055 & 0,34 & 1,2 & 8,20 & & & 0,0402 & 0,92 & & & & 4,91 & & 5,80 & 0,3 & 5,93 \\
\hline 16 & 055 & 0,35 & 1, & 8,20 & 1 & & 0,0385 & 0,81 & & 11 & 6,75 & 5,40 & & & 0,20 & 6,18 \\
\hline 17 & 054 & 0,39 & 1,4 & & & 19 & 0,03 & 0,78 & & & 6 & 5 , & & & 0 , & 6,3 \\
\hline 18 & & 0,36 & 1,4 & & & 20 & 0,04 & 0,78 & 19 & & & 5, & & 5 , & 0,07 & 5,54 \\
\hline 19 & 059 & 0,35 & 1,43 & 8,19 & 18,29 & 0,20 & 0,0417 & 0,77 & 19,02 & 11,93 & 5,88 & 4,39 & & 5,28 & 0,31 & 5,34 \\
\hline 20 & 0,059 & 0,37 & 1,38 & 8,25 & 18,34 & 0,20 & 0,0393 & 0,74 & 19,10 & 11,89 & 6,26 & 4,05 & 2,21 & 4,94 & 0,69 & 5,74 \\
\hline 21 &, 054 & 0,36 & 1,34 & 8,13 & 18,10 & 0,20 & 0,0442 & 0,60 & 18,84 & 11,75 & 5,75 & 4,65 & 1,10 & 5,54 & 0,13 & 5,33 \\
\hline 22 & 0,062 & 0,35 & 1,30 & 8,15 & 18,15 & 0,20 & 0,0464 & 0,58 & 18,88 & 12,05 & 5,10 & 3,57 & 1,53 & 4,46 & 0,46 & 4,70 \\
\hline
\end{tabular}

$h^{*}=$ Valores individuales de equivalentes en níquel del cobre, deducidos según:

$$
\left[\frac{\mathrm{Cr}_{\text {equi }}+18}{\mathrm{Ni}_{\text {equi }}+36+(\% \mathrm{Cu}) h^{*}}+0,262\right] 161-160,65=\left(\% \Delta_{\mathrm{m}}+0,89\right)
$$

$\% \Delta_{\mathrm{D}}^{\prime},=\%$ ferrita $\delta$, según DeLong, asignándole al cobre un equivalente de níquel $=0,27$

$$
\begin{aligned}
& \text { Para } \mathrm{n}=22 \\
& \bar{h}=0,27 \\
& \sigma_{\mathrm{n}-1}=0,13 \\
& L S=\bar{h}^{*}+2 \sigma=0,55 \\
& \mathrm{LI}=\bar{h}^{*}-2 \sigma=0,03
\end{aligned}
$$

$$
\begin{aligned}
& \text { Para } \mathrm{n}=21 \\
& h^{*}=0,27 \\
& \sigma_{\mathrm{n}-1}=0,10 \\
& L S=0,47 \\
& L I=0,07
\end{aligned}
$$

específicas de trabajo). Por lo tanto, toda comparación entre valores de medida de ferrita $\delta$ y valores de ferrita $\delta$ deducidos de la ecuación metalurgista del diagrama de constitución según DeLong, debe tener en cuenta este significativo dato. Así pues, para encontrar los valores individuales, colada a colada, de los equivalentes en níquel del cobre $\left(h^{*}\right)$, habrá que calcularlos haciendo que se anulen, por efecto del cobre, las diferencias entre $\Delta_{\mathrm{D}}$ (ferrita $\delta$ según DeLong) y $\Delta_{\mathrm{m}}+0,89$ (ferrita $\delta$ medida, incrementada en $0,89 \%$ por tratarse de lingotes).

En la tabla II se ofrecen los valores individuales de $h^{*}$, resultando el valor promedio de 0,29 . Rechazando el valor individual de 0,69 (correspondiente al lingote núm. 20), por superar el valor de $L S=$ 0,55 , la nueva estadística presenta un valor medio de $\bar{h}^{*}=0,27$, en cerrada aproximación con el previamente obtenido de 0,28 . 
TABLA III.- Composición química media de todos los lingotes, excluido el cobre. Valores estadísticos

TABLE III.- Average chemical composition -copper not included-from all ingots. Statistic values

\begin{tabular}{|c|ccccccc|}
\hline$n=22$ & $\mathrm{C}$ & $\mathrm{Si}$ & $\mathrm{Mn}$ & $\mathrm{Ni}$ & $\mathrm{Cr}$ & $\mathrm{Mo}$ & $\mathrm{N}$ \\
\hline$x$ & 0,057 & 0,34 & 1,40 & 8,23 & 18,28 & 0,20 & 0,0428 \\
$\sigma$ & 0,004 & 0,04 & 0,06 & 0,13 & 0,16 & 0,01 & 0,0027 \\
máx. & 0,070 & 0,39 & 1,46 & 8,73 & 18,55 & 0,21 & 0,0478 \\
mín. & 0,051 & 0,21 & 1,27 & 8,02 & 17,72 & 0,18 & 0,0385 \\
\hline
\end{tabular}

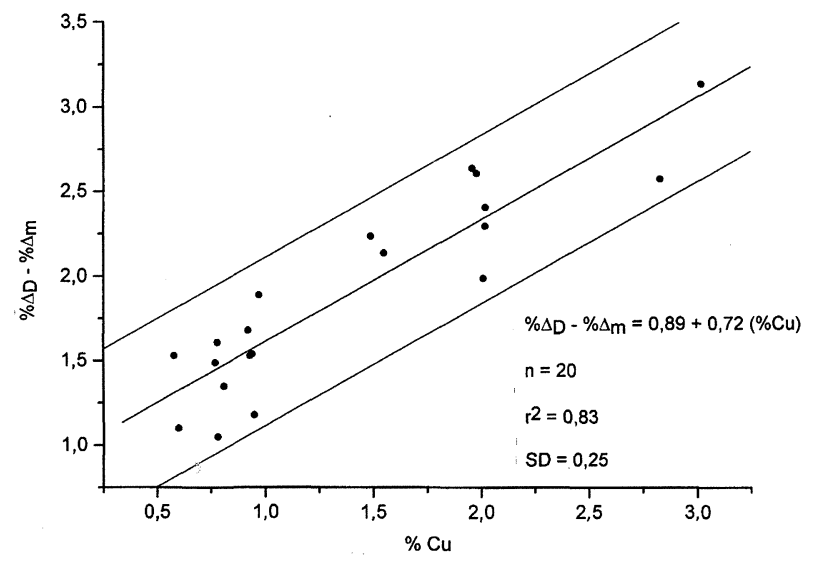

FIG. 2.- Influencia del cobre en la diferencia entre $\delta$ a partir de Delong y $\delta$ medida con ferritoscopio.

FIG. 2.- Copper influence on the difference between $\delta$ from Delong and $\delta$ measured by ferrimeter device.

Dicho valor, aplicado a cada colada para el equivalente en níquel, permite tener en cuenta el efecto gammágeno del cobre:

$$
\begin{gathered}
\mathrm{Ni}_{\text {equi }}=(\% \mathrm{Ni})+30[(\% \mathrm{C})+(\% \mathrm{~N})]+0,50 \\
(\% \mathrm{Mn})+0,27(\% \mathrm{Cu})
\end{gathered}
$$

Así, es posible recalcular los nuevos valores, según DeLong, para la ferrita $\delta$, es decir, la columna $\left(\% \Delta_{\mathrm{D}}^{\prime}\right)$ en la tabla II. Dichos valores tendrían que ser comparados con $\left(\% \Delta_{\mathrm{m}}+0,89\right)$.

La figura 3 presenta el resultado final gráfico de la correlación que tiene en cuenta el efecto gammágeno del cobre y el hecho de que la colada sea en lingotes.

\section{DISCUSIÓN}

El contenido de ferrita $\delta$ de un acero inoxidable austenítico va a depender de la composición química del mismo y de su historial termomecánico, incluyendo obviamente el modo de solidificación.

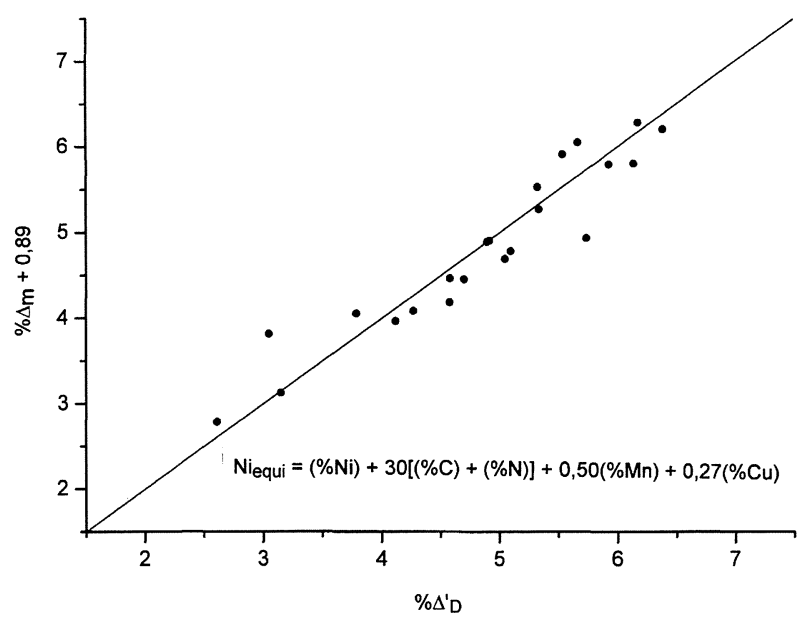

FIG. 3.- \% $\Delta^{\prime}{ }_{\mathrm{D}}$ obtenidos con la ecuación de Delong usando el equivalente de cobre. FIG. 3.- \% $\Delta^{\prime}$ obtained from Delong using
calculated copper equivalent.

Para la serie 300 de AISI, la fórmula de predicción más común suele ser el diagrama de constitución según DeLong, válido en principio para estos materiales en condiciones de solidificación de soldadura.

Este no es el caso de estos lingotes, de $40 \mathrm{~kg}$ de peso, fabricados con una cierta forma geométrica de lingotera, una determinada velocidad de colada a partir de una determinada temperatura del acero líquido y de un determinado material y espesor de pared de lingotera así como de otra serie de circunstancias que van a condicionar el modo de solidificación y la manera del enfriamiento.

Sin embargo, es necesario tener una referencia -en este caso DeLong- y hacer aproximaciones a la misma para cada contingencia de solidificación -lingotes de variadas formas geométricas, desbastes, palanquillas, etc.- que eviten la creación de todo un cuerpo de doctrina para cada variante de solidificación.

El procedimiento experimental y el estudio que se ha presentado, ponen de manifiesto que ha sido posible salvar la distancia entre ellos sobre el modo de solidificación e historia térmica con el objetivo de obtener un equivalente en níquel del cobre. Dicho equivalente está muy próximo al ofrecido por otros autores $(4,6$ y 7$)$.

\section{CONCLUSIONES}

A partir de una serie de 22 muestras de aceros inoxidables $18 \mathrm{Cr} 8 \mathrm{Ni}$, con concentraciones variables entre 0,6 y $3 \% \mathrm{Cu}$, colados en lingotes de unos 40 $\mathrm{kg}$ de peso, realizando medidas de ferrita $\delta$ con un medidor de ferrita en secciones transversales a los ejes mayores de los lingotes, se ha calculado un equivalente en níquel del cobre, que resultó ser de 0,27 , lo que permite corregir los valores según 
DeLong y obtener una buena correlación entre los valores medidos y predichos.

Este equivalente en níquel del cobre está muy próximo al que ofrecen otros autores, y permite el empleo del contenido habitual de cobre de los fundidos en el cálculo del balance austenítico, de cara a la optimización del material en comportamiento y economía.

\section{REFERENCIAS}

(1) Botella Arboledas, J. Influencia de elementos trampa en inoxidables. Informe EUR 13316 ES. Oficina de Publica- ciones Oficiales de las Comunidades Europeas, L-2985 Luxemburgo, 1991: 5, 6 y 24.

(2) ÁNGel, T.J. Iron Steel Inst., mayo 1954: 165-174.

(3) Hull, F.C. Weld. Res. Suppl., mayo 1973: 193-203.

(4) Eichelman, G.H. y Hull, F.C. Trans. ASM, 45, 1953: 77104.

(5) Ohkubo, N., Miyakusu, K., Uematsu, Y. y Kimura, H. ISIJ Intern.. 34 (9), 1994: 764-772.

(6) Ferree Jr. J.A. Patente EE.UU. núm. 3,460939. 12 agosto 1969.

(7) PotaK, M. y Sagalevich, E.A., Avt. Svarka, (5), 1972: 10-13. 